\title{
Corrigendum
}

\section{A savings index heuristic algorithm for flowshop scheduling with sequence-dependent setup times}

\author{
SR Das ${ }^{1}$, JND Gupta ${ }^{2}$ and BM Khumawala ${ }^{3}$ \\ ${ }^{I}$ George Mason University, Fairfax, VA, USA; ${ }^{2}$ The University of Alabama in Huntsville, Huntsville, AL, USA; \\ and ${ }^{3}$ University of Houston, Houston, TX,USA
}

Journal of the Operational Research Society (2004) 55, 1369. doi:10.1057/palgrave.jors.2601873

Correction to: Journal of Operational Research Society (1995) 46, 1365-1373.

In the paper, 'A savings index heuristic algorithm for flowshop scheduling with sequence-dependent setup times,' by Das et al, ${ }^{1}$ the following corrections are necessary to make the algorithm, numerical example, and computational results consistent.

1. On page 1367 please replace Equation (2) and the paragraph before that as follows:

Potential savings in setup times: Let $\pi$ be the set of all remaining $k$ jobs still to be scheduled and $e$ be the last job of partial schedule $\sigma$. Then, for any non-empty $\sigma$, it is clear that the potential savings in setup times at machine $m, R(\sigma a, m)$ can be expressed as follows:

$$
R(\sigma a, m)=\max \left\{s_{m}(e, b) ; b \in \pi\right\}-s_{m}(e, a)
$$

2. On page 1368, please change Steps 1 and 2 of the algorithm as follows:

Step 1. Consider each job $a$ in $\pi$ containing $k$ jobs. Let $e$ be the last job in partial schedule $\sigma$. Enter Step 2.
Step 2. Using Equations (2)-(5), find $S I(\sigma a)$ for each job $a \in \pi$. Augment job $a$ with maximum $\operatorname{SI}(a)$ to $\sigma$. Let $\sigma=\sigma a, \pi=\pi-\{a\}$, and $k=(k-1)$. If $k=1$, enter Step 3; otherwise return to Step 1.

3. On page 1369, Table 3, block 'At $m=2$ to job $j=$ ', column 1, row 4 , please change the entry from 207 to 407.

4. On page 1369, Table 5, the last column (for $S I(\sigma a)$ ) and row 2 (for job 3 ), please change the entry from 234 to 235.

5. On page 1369, replace the first part of line 6 after Table 5 with 'two other schedules, $(3,2,1,4)$ and $(4,2,3,1)$, each with ...'.

The authors apologize for the above errors in the original paper and are thankful to Dr Fan T Tseng of the College of Administrative Science, University of Alabama in Huntsville, Huntsville, AL, USA for discovering these errors and bringing them to our attention.

\section{References}

1 Das SR, Gupta JND and Khumawala BM (1995). A savings index heuristic algorithm for flowshop scheduling with sequence dependent setup times. J Opl Res Soc 46: 1365-1373. 